\title{
Sclerotherapy for Orbital Lymphangioma - Case Series and Literature Review
}

\author{
KARNESH CHANDRAKANT PATEL ${ }^{1}$, GEORGE KALANTZIS ${ }^{2}$, \\ NABIL EL-HINDY ${ }^{2}$ and BERNARD Y. CHANG ${ }^{2}$ \\ ${ }^{1}$ Department of Ophthalmology, Huddersfield Royal Infirmary, Huddersfield, U.K.; \\ ${ }^{2}$ Department of Ophthalmology, St. James' University Hospital, Leeds Teaching Hospitals NHS Trust, Leeds, U.K.
}

\begin{abstract}
Orbital lymphangioma is a lymphatic system lesion that commonly presents in childhood. Management of these lesions is complex. Sclerotherapy is a therapy used to treat and shrink lesions prior to or as an alternative to surgery. We present three cases of orbital lymphangioma that were treated with sclerotherapy. Case 1: A 6-month-old boy was presented in 2010 with right ptosis and proptosis. Magnetic resonance imaging (MRI) identified a lesion involving the right orbit and face. Case 2: A 3-year-old girl was presented in 2011 with intermittent right periorbital swelling and medial canthal bleeding. MRI identified a softtissue lesion in the right orbit, extending into the face. Case 3: A 3-year-old girl was presented in 2012 with vomiting, and painful right proptosis. MRI identified an intra-conal lesion in the right orbit with fluid filled levels. All three patients were treated with sclerotherapy (sodium tetradecylsulfate). Sclerotherapy is a promising treatment for orbital lymphangioma. Its use may prevent the need for, or minimise the amount of surgical management. Several sclerosants are now commonly used to treat these lesions.
\end{abstract}

Orbital lymphangioma is a rare, unencapsulated vascular malformation of the lymphatic system (composed of irregular vascular channels lined with a single layer of attenuated endothelial cells), primarily seen within the first decade of life (1). Traditional management of this entity through surgery can be challenging. This can result in partial resection, with risk of recurrence, scarring, or injury to important adjacent structures $(1,2)$. Sclerotherapy has been

This article is freely accessible online.

Correspondence to: Mr. Karnesh Chandrakant Patel, Department of Ophthalmology, Huddersfield Royal Infirmary, Huddersfield, HD3 3EA, U.K. E-mail: karnesh@doctors.org.uk

Key Words: Paediatric, orbital, lymphangioma, sclerotherapy, sodium tetradecylsulfate (STS). reported as an alternative to surgery (3). We discuss three cases treated successfully with this modality.

\section{Case Reports}

Case 1. This 6-month-old boy was referred to the oculoplastic surgeons by a paediatrician in May 2010 for a $3 \times 3 \mathrm{~cm}$ soft, non-pigmented swelling at the bridge of the nose, extending into the right upper lid. The right orbit was found to have non-axial proptosis, right upper lid ptosis, and lateral displacement of the globe. The left orbit appeared unaffected on clinical examination. Visual acuity appeared to be unaffected in both eyes, but this was difficult to confirm due to the patient's age.

Magnetic resonance imaging (MRI) of the head and orbits in September 2009 identified a substantial soft tissue enhancing lesion involving the right scalp and upper lid, extending into the extraconal space both superiorly and medially, almost to the orbital apex (Figure 1). The lesion extended downwards through the right maxilla to the hard palate (Figure 2). In addition, a large loculated cyst was found in the right intraconal space surrounding the optic nerve/sheath complex. The right optic nerve appeared normal, and the lesion did not appear to extend into the right cavernous sinus.

After discussion in a multi-disciplinary team meeting, it was felt that lymphangioma was the likely primary diagnosis.

The patient underwent patching for stimulus deprivation amblyopia in the right eye in June 2012 as visual acuity had decreased from 0.0 to 0.4 on Logarithmic Minimal Angle of Resolution (LogMAR) chart measurement since March 2012. The left eye remained unaffected at 0.0 LogMAR.

The patient was simultaneously referred to a specialist centre for sclerotherapy in June 2012, and subsequently underwent three courses of sclerotherapy using sodium tetradecylsulfate in the orbit and doxycycline in the face between 2012 and 2013 .

The patient was left with minimal changes in the orbit on repeat MRI, although a solid microcystic component 
remained in the right brow, medial canthus, and forehead. The patient underwent further right supraorbital sclerotherapy in November 2014, using OK-432 (Picibanil; Chugai Pharmaceutical Co Ltd). Repeat ultrasound showed some reduction in the facial microcystic components. The patient has now been followed up for over 2 years since their last treatment, and their condition has remained stable. Visual acuity has remained stable at $0.02 \log$ MAR in the left eye. The right eye has stablised at $0.5 \mathrm{LogMAR}$.

Case 2. This 3-year-old girl was presented in December 2011 with longstanding variable right periorbital swelling associated with intermittent bleeding from the medial canthus over the previous month. Examination found nonaxial proptosis, causing lateral globe displacement. Visual acuity was 0.1 LogMAR in both eyes.

T2-Weighted MRI of the head was performed, identifying a soft-tissue lesion medial to the right orbit, extending to above the right globe (Figure 3 ). The lesion also extended extra-orbitally.

The patient initially underwent surgery with right transconjunctival orbitotomy and orbital debulking in May 2012. This failed to improve symptoms, and further MRI of the head showed subsequent recurrence in the right maxillary area and eyelids. The patient was referred to a specialist centre for sclerotherapy, and underwent two courses of sclerotherapy using sodium tetradecylsulfate in early 2013.

By 4 months' post-treatment, there was significant improvement seen clinically and on repeat MRI scanning. There was no further bleeding from the medial canthus. Subsequent ultrasound scan in November 2013 showed there was still some deep tissue involvement in the right cheek; this remains under surveillance. Visual acuity is currently Snellen chart 6/6 in both eyes. The patient's status has remained stable over the 3 years since the last treatment.

Case 3. This 3-year-old girl was presented in May 2012 with a 2-day history of headaches, vomiting and swelling of the right upper lid. She was found to have restriction of right extra-ocular movements in all directions, associated with a $3 \mathrm{~mm}$ axial proptosis. There was no evidence of optic neuropathy. The left side was normal. Visual acuity was 0.0 on LogMAR in both eyes.

Computerised tomographic scan of the head identified a right intra-conal lesion, extending into the eyelids (Figure 4).

The patient was referred directly to a specialist centre in July 2012 for sclerotherapy. The patient underwent three sclerotherapy procedures (December 2012, February 2013, and May 2013) with sodium tetradecylsulfate.

The patient showed a good response to treatment, and extra-ocular muscle restriction, proptosis and upper lid swelling all resolved. Only a small 4-mm fluid-filled cyst remained in the posterior orbit on MRI. The patient has been

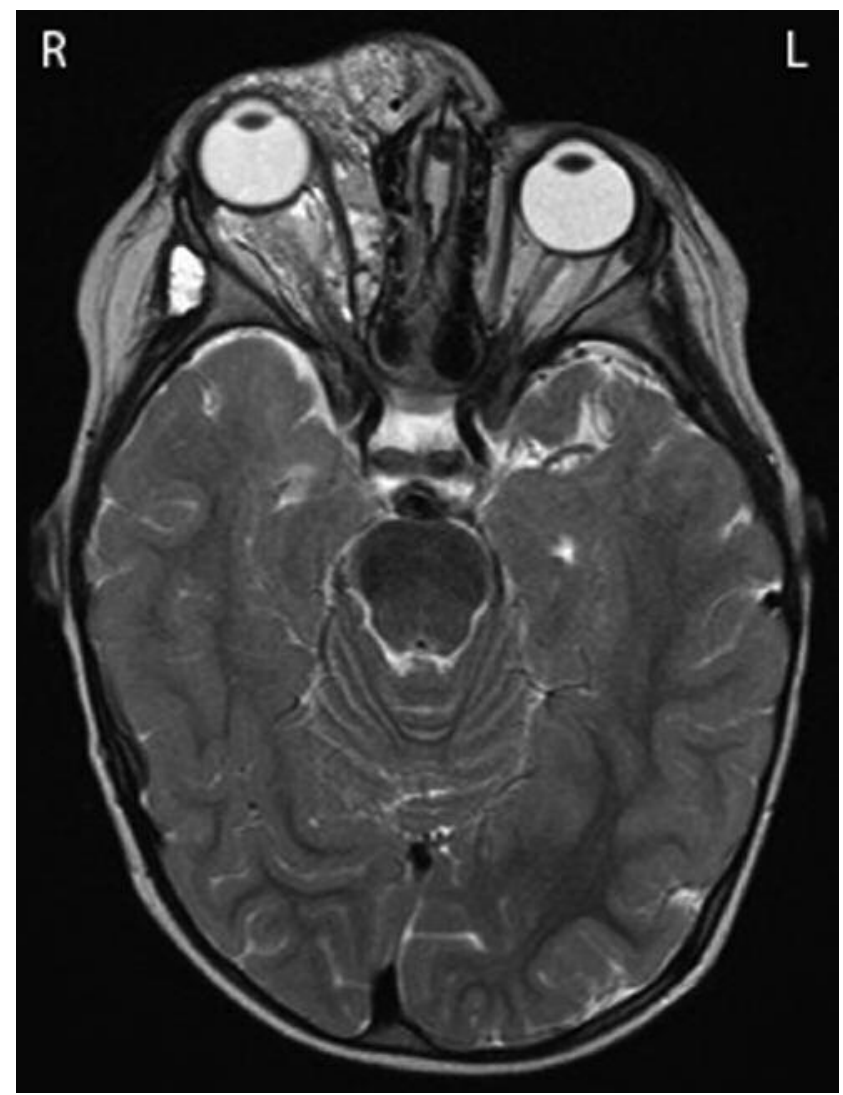

Figure 1. Case 1: T2-Weighted magnetic resonance imaging showing a lesion involving the right upper lid, extending into the extra and intraconal spaces medially, almost to the orbital apex.

observed now for over 3 years without any further issues. Visual acuity has remained stable at 0.0 LogMAR.

\section{Discussion}

Approximately $25 \%$ of orbital lymphangioma cases involve the orbit (2). Previously reported signs on presentation include progressive proptosis (axial or non-axial), ptosis, ophthalmoplegia, and strabismus $(2,3)$. Previously reported symptoms include pain, diplopia, and visual loss (4). Intraorbital haemorrhage has been reported as a common cause of exacerbation of proptosis. Such haemorrhage was spontaneous, or after local/systemic infection, or local trauma $(2,5,6)$.

Saha and Leatherbarrow consider orbital lymphangiomas to be abnormal channels lined with endothelial cells (7), instead of truly being of lymphatic channel origin, since such channels do not normally exist in the orbit (3).

Orbital lymphangiomas are currently classified as Type 1 (low flow and no vascular system connection) orbital vascular malformations by the International Orbital Society. 


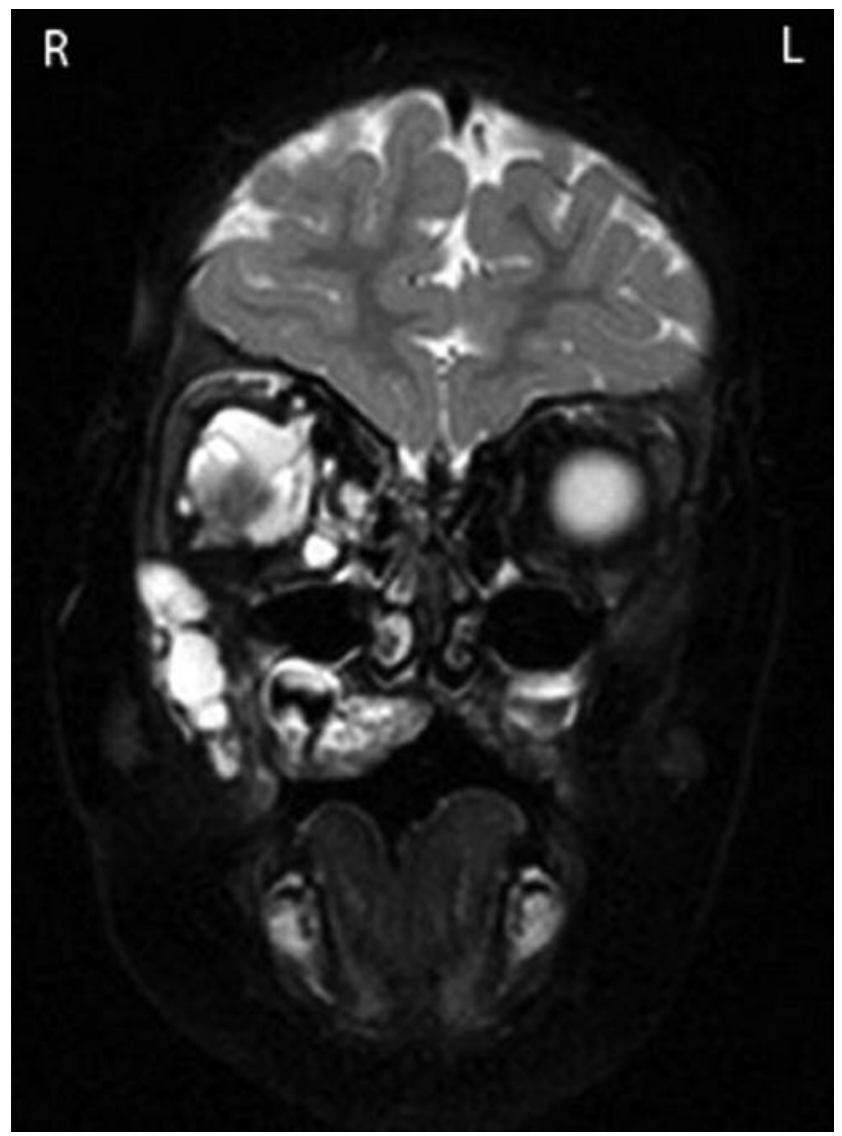

Figure 2. Case 1: T2-Weighted magnetic resonance imaging showing the lesion extending downwards towards the hard palate.

Any lesions that are found to have any vascular system connection fall under Type 2 (7).

Management of orbital lymphangioma can be difficult. Due to the age of most patients at presentation, maintaining good visual acuity and preventing amblyopia are significant factors that help guide treatment. Treatment can be split into conservative, surgical, and non-surgical management.

Imaging with MRI can be useful to plan management. Although computed tomography allows assessment of the bony orbit and surrounding facial bones, this is not useful for planning sclerotherapy. Ultrasound scans are useful for assessing the orbit post-treatment (8).

Harris et al. advocated conservative management because of the complex spread of such lesions and the risks both of bleeding, and of potentially damaging adjacent structures when attempting to excise the lesion (1). However, this was before sclerotherapy use was a definitive option, and nowadays, conservative management is not generally suggested (7).

Surgical excision is challenging, since orbital lymphangiomas are unencapsulated. Thus, in some cases only partial excision is performed to debulk the lesion and

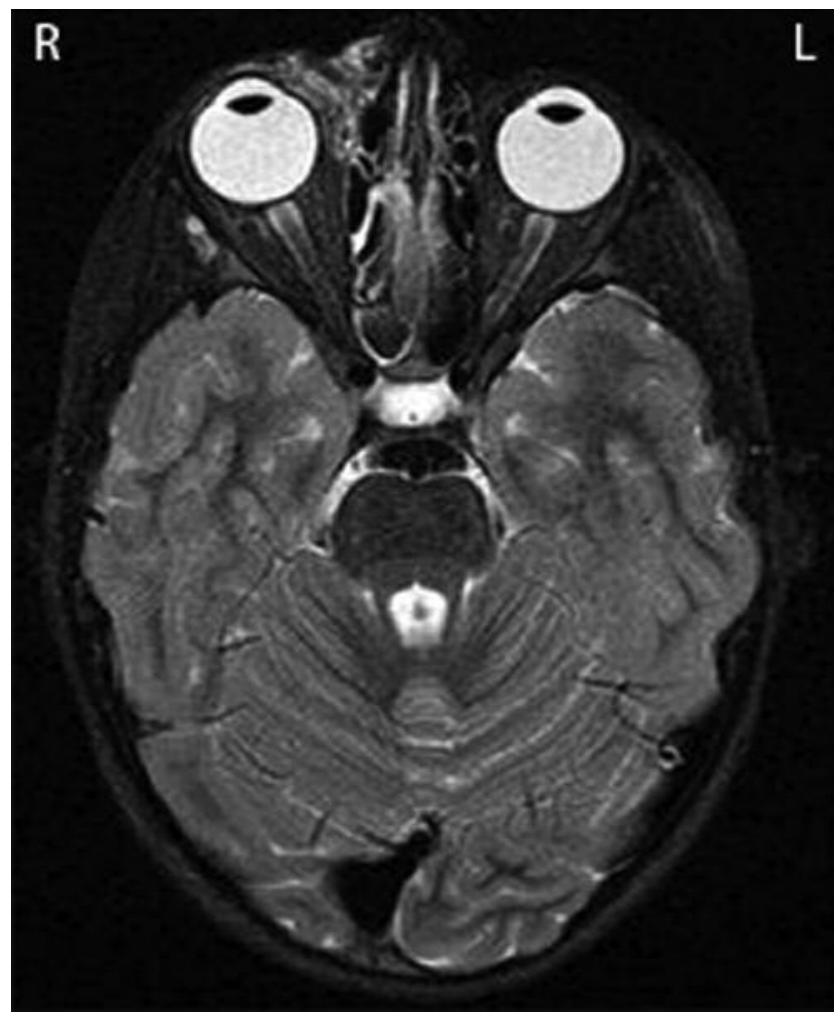

Figure 3. Case 2: T2-Weighted magnetic resonance imaging showing a lesion medial to the right orbit, extending into the right orbit.

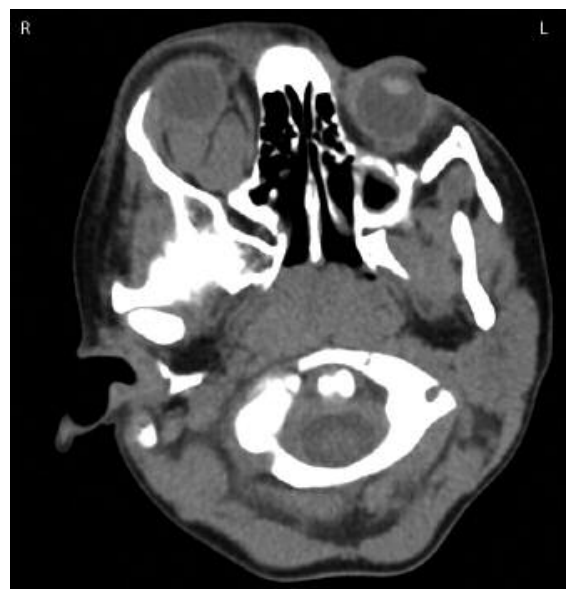

Figure 4. Case 3: Computed tomographic scan showing a right intraconal lesion, with right upper lid soft-tissue involvement.

reduce the mass effect it causes $(1,7)$. The risk of intraoperative haemorrhage is significant, but can be reduced 
using diathermy, or carbon dioxide laser $(5,7)$. Boulos et al. have reported injecting fibrin glue into cystic components of lesions during excision to stabilise the structures being removed. Saha and Leatherbarrow have also used this approach (although it should be noted it is not licensed for that specific use) $(7,9)$.

Sclerosants have been used for treating lymphatic malformations for several years. Agents that have been previously reported as being used for orbital lymphangioma include OK-432 (Picibanil; Chugai Pharmaceutical Co Ltd), sodium tetradecylsulfate (multiple manufacturers), 5\% sodium morrhuate, and bleomycin A5 (10-14).

Percutaneous drainage and ablation of cystic components has also been advocated for orbital lymphangiomas, using a combination of intra-cyst injections of either sequential sodium tetradecylsulfate and ethanol for macrocysts $(>1 \mathrm{~cm})$, or doxycycline for microcysts $(<1 \mathrm{~cm})(15)$.

Management of orbital lymphangioma continues to require a multi-disciplinary approach, and early involvement of other specialities when needed can prevent delays in arranging imaging or treatment. It is not unusual nowadays for treatment to comprise of both surgical and non-surgical aspects in order to remove/shrink as much of the lesion as possible while minimising visual and functional loss. It is important to remain in frequent communication with the patient and/or their parents, as well as colleagues, to ensure a consistent message and plan of action. What is clear is that sclerotherapy is now an established management option for these lesions, with good results (and minimal complications) in appropriately chosen patients (8).

\section{References}

1 Harris GJ, Sakol PJ, Bonavolontà G and De Conciliis C : An analysis of thirty cases of orbital lymphangioma. Pathophysiologic considerations and management recommendations. Ophthalmology 97(12): 1583-1592, 1990.

2 Jones IS: Lymphangiomas of the ocular adnexa: an analysis of 62 cases. Trans Am Ophthalmol Soc 57: 602-665, 1959.

3 Iliff WJ and Green WR: Orbital lymphangiomas. Ophthalmology 86(5): 914-929, 1979.
4 Malhotra AD, Parikh M, Garibaldi DC, Merbs SL, Miller NR and Murphy K: Resection of an orbital lymphangioma with the aid of an intralesional liquid polymer. Am J Neuroradiol 26(10): 2630-2634, 2005.

5 Tunç M, Sadri E and Char DH: Orbital lymphangioma: an analysis of 26 patients. Br J Ophthalmol 83(1): 76-80, 1999.

6 Graeb DA, Rootman J, Robertson WD, Lapointe JS, Nugent RA and Hay EJ: Orbital lymphangiomas: clinical, radiologic, and pathologic characteristics. Radiology 175(2): 417-421, 1990.

7 Saha K and Leatherbarrow B: Orbital lymphangiomas: a review of management strategies. Curr Opin Ophthalmol 23(5): 433438, 2012.

8 Barnacle AM, Theodorou M, Maling SJ and Abou-Rayyah Y: Sclerotherapy treatment of orbital lymphatic malformations: a large single-centre experience. Br J Ophthalmol 100(2): 204208, 2016.

9 Boulos PR, Harissi-Dagher M, Kavalec C, Hardy I and Codère F: Intralesional injection of Tisseel fibrin glue for resection of lymphangiomas and other thin-walled orbital cysts. Ophthal Plast Reconstr Surg 21(3): 171-176, 2005.

10 Ogita $\mathrm{S}$, Tsuto $\mathrm{T}$, Tokiwa $\mathrm{K}$ and Takahashi $\mathrm{T}$ : Intracystic injection of OK-432: a new sclerosing therapy for cystic hygroma in children. Br J Surg 74(8): 690-691, 1987.

11 Suzuki Y, Obana A, Gohto Y, Miki T, Otuka H and Inoue Y: Management of orbital lymphangioma using intralesional injection of OK-432. Br J Ophthalmol 84(6): 614-617, 2000.

12 Poonyathalang A, Preechawat P, Jiarakongmun P, Pongpech S: Sclerosing therapy for orbital lymphangioma using sodium tetradecyl sulfate. Jpn J Ophthalmol 52(4): 298-304, 2008.

13 Schwarcz RM, Ben Simon GJ, Cook T and Goldberg RA: Sclerosing therapy as first line treatment for low flow vascular lesions of the orbit. Am J Ophthalmol 141(2): 333-339, 2006.

14 Yue H, Qian J, Elner VM, Guo J, Yuan YF, Zhang R and Ge Q: Treatment of orbital vascular malformations with intralesional injection of pingyangmycin. Br J Ophthalmol 97(6): 739-45, 2013.

15 Hill RH 3rd, Shiels WE 2nd, Foster JA, Czyz CN, Stacey A, Everman KR and Cahill KV: Percutaneous drainage and ablation as first line therapy for macrocystic and microcystic orbital lymphatic malformations. Ophthal Plast Reconstr Surg 28(2): 119-125, 2012

Received November 12, 2016

Revised February 2, 2017

Accepted February 7, 2017 